\title{
Glomerulonephritis, Pathogenetic Mechanisms and Therapeutic Options: An Overview
}

Gaspare Elios Russo ${ }^{*}$, Tania Gnerre Musto ${ }^{2}$, Massimo Testorio ${ }^{2}$, Alessio Molfino ${ }^{1}$, Andrea Martinez ${ }^{2}$, Alessandra Nunzi ${ }^{2}$, Virgilio DeBono ${ }^{2}$, Dmytro Grynyshyn ${ }^{2}$, Annarita D'Angelo ${ }^{2}$, Georgie Innico ${ }^{2}$ and S. Lai ${ }^{1}$

${ }^{1}$ Department of Clinical Medicine, Sapienza University of Rome, Italy

${ }^{2}$ Department of Urologic, Obstetrical and Gynecological Sciences, Sapienza University of Rome, Italy

*Corresponding author: Gaspare Elios Russo, Department of Clinical Medicine, Sapienza University of Rome, Italy, Tel: (407) 996-4282; E-mail: gasparelios.russo@uniroma1.it

Rec date: June 11, 2014, Acc date: June 28, 2014, Pub date: July 5, 2014

Copyright: ( 2014 Russo GE, et al. This is an open-access article distributed under the terms of the Creative Commons Attribution License, which permits unrestricted use, distribution, and reproduction in any medium, provided the original author and source are credited.

\begin{abstract}
Introduction: Most forms of human glomerulonephritis (GN) result from immunologic mechanisms that are mediated by the actions of multiple elements of both the innate and adaptive immune systems, thereby resulting in different clinical manifestations. The treatment of immune-mediated kidney disease is based on steroids and immunosuppressive drugs that interfere with the immune processes. These groups of drugs have led to significant benefits, but severe side effects are still frequent. Monoclonal antibodies directed against molecules of inflammation or several cellular components have emerged in clinical practice. Plasmapheresis and new methods to reduce the risks associated with the procedure with standard therapies may be combined. Moreover new therapeutic options have been proposed, as the use of natural anti-inflammatory cytokines or intracellular signaling reducing inflammation.
\end{abstract}

Materials and Methods: We conducted a systematic review on the pathogenetic mechanisms of glomerulonephritis and their therapies.

Results: We analized all RCTs and quasi-RCTs evaluating the current knowledge about pathogenetic mechanisms of glomerulonephritis and related therapy were considered.

Conclusion: The pathogenetic mechanisms of glomerulonephritis are complex and strongly influenced by immunogenetic factors. Several clinical trials to identify the best therapeutic options for glomerulonephritis have been conducted, but currently, although significant advancements over the last 10 years have been obtained, important questions are still unanswered. Moreover, we need to consider many and important side effects that have the main therapies for glomerulonephritis. Therefore the development of a web enabled data base to assist nephrologists for the treatment of patients with glomerulonephritis is strongly suggested.

Keywords: Glomerulonephritis; Therapy; Plasmapheresis; Nanotechnology

\section{Introduction}

\section{Pathogenesis of glomerulonephritis}

A large body of clinical, immunological, and experimental data supports the hypothesis that most forms of human glomerulonephritis (GN) result from immunologic mechanisms [1]. The etiology in human GN is largely unknown with the exception for infectious agents, such as B-hemolytic streptococcus in poststreptococcal GN, or hepatitis $\mathrm{C}$ virus in cryoglobulinemic membranoproliferative glomerulonephritis (MPGN). Many agents cause GN by inducing loss of tolerance to self-antigens and mechanisms like epitope spreading and molecular mimicry, rather than by a direct immune response to a foreign etiologic agent, as experimentally-observed with serum sickness. The pathogenetic mechanisms of glomerulonephritis are numerous and complex, involving both humoral and cell-mediated immune responses. The nature of the immune responses which leads to GN are strongly influenced by immunogenic phenotypes. The most common cause of glomerulonephritis is deposition of immune deposits (antigen-antibody complexes) to the level of the glomerular basement membrane. These may be linear (disease by anti-basement membrane) or granular deposits on the capillary wall (sub-endothelial deposits, as in the mesangium-capillary glomerulonephritis type I) or in the mesangium (IgA glomerulonephritis) or also on the epithelial side of the basement membrane (membranous glomerulonephritis) [2]. Deposition of immune complexes is followed by an inflammatory response with activation of the cascade of the complement factors $[1,3]$. Indeed, there is a severe inflammation, as occurs in vasculitis (ANCA positive necrotizing glomerular nephritis) [4] without the deposition of immune complexes. When inflammatory changes occur, for example, glomerular hypercellularity is due to infiltrating hematopoietic cells (such as neutrophils and macrophages), proliferating glomerular cells or both. These effector cells induce other abnormalities, such as thrombosis, necrosis and crescent formation that may result in rapidly progressive renal insufficiency and nephritis. By comparison, non-inflammatory lesions resulting from immune injury usually involve the podocyte as the major effector cell and are associated with a major functional change of the glomerulus (usually an increase in protein permeability), but without light microscopic 
evidence of damage. This event most commonly results in proteinuria and in the nephrotic syndrome and may lead to glomerular sclerosis [1]. The nephritogenic immune response exhibits both humoral and cellular components. In both cases, macrophages and T lymphocytes play a key role: macrophages activated by $\mathrm{T}$ cells release inflammatory substances (cytokines, oxidizing substances, etc.) and trigger the processes that favor fibrosis. The humoral or T helper cell 2 (Th2)regulated, immune response leads to immunoglobulin deposition formation and complement activation in glomeruli. The cellular or $\mathrm{T}$ helper cell 1(Th1)-regulated, immune response contributes to the infiltration of glomeruli by circulating mononuclear inflammatory cells (including lymphocytes and macrophages) [1,5] and crescent formation. The choice of the drug in the treatment of glomerular nephritis is influenced by the pathogenic mechanisms and therefore should be an informed decision.

\section{Treatment of Glomerulonephritis}

The treatment of GN is still empirical and aimed to: $[6,7]$.

- Reduce blood pressure, especially if the patient with proteinuria

- Reduce the proteinuria, by ACE or Angiotensin (ANG II) inhibitors [7-9].

- Use steroids (membranous GN, focal glomerulosclerosis, IgA nephropathy deposits) [10-12].

-Use immunosuppressants (cyclosporine and mycophenolate), often with no defined guidelines.

The treatment of GN should be mainly based on the use of molecules that interfere with the immune mechanisms that determine kidney disease [13]. The classes of drugs historically used are steroids, immunosuppressive and calcineurin inhibitors associated with plasmapheresis [1].

\section{Steroid therapy}

Corticosteroids are involved in a wide range of physiological processes, including stress response, immune response, and regulation of inflammation, carbohydrate metabolism, protein catabolism, blood electrolyte levels, and behavior. Corticosteroids are powerful antiinflammatory drugs used to suppress the effects of the immune response of any origin: autoimmune diseases, allergy or transplant rejection. One of the most commonly used is prednisone, synthetic analogue of cortisol presenting mineralocorticoid action in addition to the glucocorticoid effect [14]. In plasma, cortisol is linked to corticosteroid binding protein $(\mathrm{CBG})$ and albumin, and prednisone circulates free or weakly bound to albumin. The important antiinflammatory and immunomodulatory effects of GCs are predominantly mediated by genomic mechanisms. Glucocorticoids, as lipophilic substances, passively diffuse through the cell membrane and bind the glucocorticoid receptor binding (GBR), ubiquitously expressed, ultimately inducing ("transactivation") or inhibiting ("transrepression") the regulator proteins synthesis [15,16]. The mechanisms of action of steroids depend from the dosage used. Higher dosages increase GBR saturation in a dose-dependent manner, which intensifies the therapeutically relevant genomic actions. Increasing dosages induce additional and qualitatively different non-specific nongenomic effects of GCs. When used at high doses, the complex binds to DNA sequences named Glucocorticoid Responders Elements (GRE), facilitating or inhibiting the assembly of proteins and initiating transcription of RNA polymerase II [16,17]. At a lower dose, corticosteroids block the transcriptional activity of Nuclear Factor $\mathrm{kB}$ (NF- KB) inhibiting the production of inflammatory cytokines, such as TNF-alpha and IL- 1, adhesion molecules and complement factors. The genomic action is slow and significant changes in regulator protein concentrations before 30 minutes are not observed because of the time required for GBR activation/translocation, transcription, and translation effects $[18,19]$. The third is the "Non-genomic mechanisms of action", which is represented by the direct activation of enzymes cascade through second messengers and arise within a few seconds or minutes. These effects justify the rapidity of the pharmacological response which is not related to interference with the genes transcription. High doses of steroids lead to a direct cytolytic effect of $\mathrm{T}$ and $\mathrm{B}$ cells. This is the rationale for the use of high doses of steroid in rapidly progressive glomerulonephritis, where the timeline of therapy is crucial and the use of cortisone is a common practice. The direct effects of steroids on transcription are mediated by the reduction of the synthesis of IL-8, which is responsible for the migration of neutrophils into sites of inflammation, by binding and blocking of the promoter of IL- $1 \alpha$ and IL- $\beta$ synthesis and inhibition of the synthesis of most of the pro-inflammatory cytokines. The use of the steroids is associated with the presence of a neutrophil leukocytosis due to the decrease of migration into sites of inflammation and the inhibition of apoptosis. The human glucocorticoid receptor gene is located on chromosome 5q 31-32 [20], but some variants in humans exist, so it is possible to observe the expression of both the GRP $a$ and $\beta$. This genetic heterogeneity, by altering the metabolic processes, can provide the rationale of the extreme variability of the effects of steroids that can be observed. In vitro studies demonstrated that, when type B cells are more expressed, there is a greater resistance to glucocorticoids. Furthermore, pro-inflammatory molecules such as cytokines TNF $\alpha$ and IL-1 have the ability to up-regulate the receptor $\beta$ in cells during inflammation. Daily administration of a single dose of prednisone does not suppress the cortisol circadian peak on the next morning; however, the toxicity may still occur in long-term therapies for interference with the hypothalamic-pituitary-adrenal axis [21]. The side effects can be numerous and very serious and occur when patients receive more than $20 \mathrm{mg}$ per day, for more than three weeks. High blood pressure that is observed in patients may be related to increased fluids and sodium retention or an excessive response to angiotensin II for overexpression receptors. Steroid-induced osteoporosis may be a side-effect of long-term corticosteroid use. Indeed, the GRE inhibits osteocalcin transcription in the osteoblasts. Osteocalcin is a major extracellular matrix protein that promotes bone mineralization. By suppressing immune reactions steroids may cause infections. Glucocorticoids promote gluconeogenesis and lipolysis with an increased susceptibility to hyperglycemia, insulin resistance and diabetes mellitus. Chronic use of glucocorticoids may predispose to cataract and retinopathy. The negative effects of steroids use can be devastating in children causing growth retardation. Especially in children and in older adults it should be considered the possible development of neuropsychiatric disorders [22]. The rapid suppression of the hypothalamic-pituitary adrenal insufficiency can result in clinically significant side-effects when the drug is suddenly stopped or during stress. Therefore, in the case of suspension, the drug should be gradually reduced observing caution. Considering these several side effects, steroids should be administered at the lowest dose for the shortest time possible, considering, however, that a suspension with an early recurrence of the disease can often occur. In the future it is hoped to use elective glucocorticoids modulating specific transcriptional effects [23]. 


\section{Immunosuppressive therapy}

Immunosuppressant drugs present different modalities of action. Particularly, the alkylating agents alter DNA, azathioprine is an antimetabolite and mycophenolate mofetil acid prevents the synthesis of purines. The final effect is the reduction in cell reproduction and in particular in the release of cytokines and chemokines. Immunosuppressive therapy has led to excellent results in the treatment of kidney immune diseases. This is highlighted by the excellent clinical results obtained with steroids use in lupus nephritis, especially with the introduction of high-dose steroid boluses and / or cyclophosphamide. Excellent results were also achieved with the use of cyclophosphamide in ANCA-associated necrotizing vasculitis [24,25]. However, the limitations of immunosuppressive therapy are still present. The immune-mediated diseases of the kidney are due to the coexistence of several pathogenic mechanisms and the use of drugs with different actions is justified by their ability to interfere with the various mechanisms of the immune system [26].

\section{Alkylating agents}

Alkylating agents are compounds able to insert alkyl groups within the macromolecules of organic compounds such as, for example, proteins and DNA. These agents cause mismatches and breakage inside of the filaments, which can generate errors during cell replication and determine a "genotoxic effect" with enhanced toxicity in cell reproduction [27]. B lymphocytes and, to a lesser extent, T lymphocytes are particularly sensitive to these agents. Cyclophosphamide (CYC) is the alkylating agent used for most inflammatory disorders. Chlorambucil is used in patients who cannot tolerate CYC, particularly in those who develop bladder toxicity. The ability of these drugs to interfere with cell reproduction provides the rationale for their use in the therapy of diseases associated with cell proliferation, as occurs in proliferative glomerulonephritis intra and extra capillaries. It is a prodrug that is converted by liver cytochrome P450 (CYP) enzymes to its active form, the metabolite 4-hydroxy cyclophosphamide, mustard fosforamide, which interferes with DNA synthesis by blocking replication and transcription [28]. The 4hydroxy-cyclophosphamide is rapidly converted to its tautomer aldofosfaramide and both of these metabolites diffuse passively outside of hepatocytes, circulate in the plasma and enter passively into all other cells. The aldofosfaramide is an unstable compound that, through a non-enzymatic reaction, produces fosfaramide mustard and acrolein, which is mainly responsible for the toxicity bladder. In plasma, the active metabolites are linked to plasma proteins and are distributed in all tissues, including the brain and spinal cord, cross the placenta and are also found in breast milk. Both active and inactive metabolites are excreted through the urine in about 48 hours and the dose should be appropriate to the degree of renal function. It is the mustard fosfaramide to be clinically responsible for the pharmacological effects; therefore, the ability to generate intracellular mustard fosfaramide from aldofosfaramide is fundamental to achieve the therapeutic result. The cell is equipped with its own detoxification mechanisms. The complexity of these mechanisms of production of active metabolites from the drug administered, together with sophisticated systems of organism detoxification, justify the interindividual variability of therapeutic response and side effects. In addition, some differences could be due to polymorphism in the CYP enzymes. There are numerous studies on the association between CYP3A4 and 3A5 genotypes and response or survival in patients treated with cyclophosphamide [29]. Cyclophosphamide has severe and life-threatening adverse effects, including acute myeloid leukemia, bladder cancer, hemorrhagic cystitis, and permanent infertility, especially at higher doses. Cyclophosphamide, in fact, causes bone marrow suppression in a dose-dependent manner. Patients should be subjected to a control of complete bi-weekly, and the dose should be adjusted to number of white blood cells, whereas the granulocytopenia in the context of glomerulonephritis is not a purpose of the therapy. Lymphopenia is accentuated by concomitant steroid therapy, and in the course of lupus nephritis, must be differentiated from recurrence of the disease. Typically, the toxic effect on other components of the bone is less and anemia, and thrombocytopenia are less frequent. In order to prevent side effects due to the renal elimination of the drug and its metabolites, it is recommended hydration and concomitant administration of mesna (sodium 2 mercaptoetano sulfonate), which binds acrolein inactivating them. It can be administered either orally or intravenously, both effective in preventing the onset of hemorrhagic cystitis and in reducing the incidence of cancers of the bladder. Oral absorption is rapid. Oral administration usually corresponds to daily dosing and intravenous use to intermittent dosing (eg, every three to four weeks), but some exceptions exist. For example, extremely ill patients who are unable to ingest medications orally may receive daily doses of CYC via the intravenous route, at the same doses they would otherwise receive orally. It is useful as a preventive administration of CYC in the morning, to avoid stagnation of the drug in the bladder. Other common side effects are nausea, hair loss and hyponatremia for inappropriate $\mathrm{ADH}$ secretion. These effects generally occur for the higher doses of $30-40 \mathrm{mg}$ per day. Sometimes, it is necessary to consider antibiotic therapy with trimethoprim-sulfamethoxazole for prophylaxis of Pneumocystis infections cute, in patients at risk [30].

\section{Antimetabolites}

\section{Azathioprine}

Azathioprine is a purine analogue and is administered as a prodrug of 6-mercaptopurine, which is an inhibitor of purine synthesis. The active molecule interferes with DNA synthesis by blocking the de novo formation of the purines, with the final result of inhibiting cell proliferation. The $\mathrm{T}$ and $\mathrm{B}$ lymphocytes are particularly sensitive to blockade of purines, as they have no alternative enzymatic pathways "rescue ". It follows a block both cell-mediated immunity that humoral immunity, with a reduction of the production of antibodies [31]. Azathioprine (AZA) is a prodrug, that is well absorbed from the gastrointestinal and is quickly converted by $90 \%$ to 6 -mercaptopurine (6-MP) by a nonenzymatic nucleophilic attack by sulfhydrylcontaining compounds, such as glutathione or cysteine, present in red blood cells and other tissues. 6-mercaptopurine is then metabolized in the liver and enzymatically converted into 6 tiourico acid by xanthine oxidase and Thiopurine-S-methyltransferase. Therefore, the plasma levels of catabolites of azathioprine may increase, if it is simultaneously administered allopurinol, which blocks xanthine oxidase. In these cases, it should be a reduction in the dose of azathioprine in order to prevent toxic effects. The therapeutic efficacy, bone marrow toxicity, and liver toxicity of azathioprine and 6-mercaptopurine may be related to their metabolites: 6-thioguanine and 6methylmercaptopurine. Toxic effects of azathioprine and its metabolites are primarily in the bone marrow with leukopenia, thrombocytopenia and anemia and will be more susceptible to infection; regular monitoring of the blood count is recommended during treatment. Nausea and vomiting are common adverse effects, especially at the beginning of a treatment. Side effects that are 
probably hypersensitivity reactions include dizziness, diarrhea, fatigue, and skin rashes. Excretion is mainly renal and is also deleted a portion of mercaptopurine and drug unchanged. Azathioprine, in the context of immune renal diseases, is mainly used in the phases of maintenance rather than in the induction therapy, where higher doses are required and therefore more toxic drug. Its use is still burdened with significant toxicity, so that, in recent years, its use was reduced. The comparison studies, performed both in ANCA associated vasculitis that in lupus glomerulonephritis, including AZA and mycophenolate mofetil (MMF) have reported equivalent results in terms of effectiveness, but most of the toxicity of AZA compared with MMF [1,32-36].

\section{Mycophenolate mofetil}

Mycophenolate mofetil is a powerful inhibitor of lymphocyte proliferation. The drug is rapidly hydrolyzed after absorption to mycophenolic acid, its active metabolite, which is a potent, selective non-competitive reversible inhibitor of the enzyme inosine monophosphate dehydrogenase (IMPDH) and, therefore, inhibits the conversion of hypoxanthine guanine, which cannot be incorporated into DNA. The selective inhibition of de novo synthesis of purines is critical for the proliferation of $\mathrm{B}$ and $\mathrm{T}$ lymphocytes. Only free mycophenolic acid is believed to be pharmacologically active. In general, 97 percent of mycophenolic acid is bound to albumin. This percentage is decreased in the setting of hyperbilirubinemia, therefore, the levels of free mycophenolic acid, may be increased in patients with hepatic dysfunction or hypoalbuminemia. The pharmacological effects are multifarious [1]. The MPA suppresses the formation of antibodies by $\mathrm{B}$ lymphocytes, prevents the glycosylation of glycoproteins of lymphocytes and monocytes that are involved in adherence to endothelial cells and intercellular could inhibit the recruitment of leukocytes at sites of infection and inflammation and in the rejection of transplantation. MMF, therefore, does not inhibit early events of activation of mononuclear cells, such as the production of IL-1 and IL-2, but blocks these events through interference with DNA synthesis and proliferation of lymphocytes. Although therapy with MMF is associated with side effects that must be recognized and treated promptly [37,38]. Measurement of free and total mycophenolic acid in the serum as a guide to dose adjustment has been advocated by some investigators but is not a common practice. These effects can occur during treatment such as gastrointestinal symptoms (vomiting, diarrhea, and nausea), opportunistic infections (especially urinary tract), leukopenia, and, rarely, anemia. It is important; therefore, also in this case, patients undergo clinical controls, with particular attention to the values appropriate blood. Among the most common side-effects of this drug is an increased blood cholesterol level. Other changes in blood chemistry such as hypomagnesemia, hypocalcemia, hyperkalemia, and an increase in BUN are regularly noted. Progressive multifocal leukoencephalopathy is a usually fatal infection of the brain that occurs rarely with this and other immunosuppressive drugs. The overall pharmacological effect of MMF, which consists in a reduction of cell replication, has widespread and privileged the use of the drug in the forms associated with inflammatory phenomena such as proliferative lupus nephritis. However, MMF is also used in the forms of glomerulonephritis with nephrotic syndrome where you can get the clinical results [1,37]. In these contexts, its effect was comparable to that of cyclophosphamide, but encumbered with fewer side effects [38].

\section{Cyclosporin A}

Cyclosporin A is a lipophilic cyclic nonribosomal peptide consisting of 11 amino acids and is a drug that reduces the activity of the immune system by interfering with the activity and growth of $\mathrm{T}$ cells mediated response [1,39]. Ciclosporin binds to the cytosolic protein cyclophilin (immunophilin) of lymphocytes, especially $\mathrm{T}$ cells. This complex of ciclosporin and cyclophilin inhibits calcineurin, which is responsible for activating the transcription of interleukin 2. In T-cells, activation of the T-cell receptor normally increases intracellular calcium, which acts by calmodulin to activate calcineurin [40]. They are mainly involved in both Th1 cells $\mathrm{T} 1$ suppressor. The particular sensitivity of $\mathrm{T}$ lymphocytes in this molecule is linked to the low amount of calcineurin expressed in these cells which favors the complete inhibition. This particular action on T lymphocytes makes it useful in the use of cyclosporine regimens in combination with other immunosuppressants, as often happens for lupus nephritis. Cyclosporine also inhibits lymphokine production and release of other interleukins such as TNFa, IL- 3, IL-4 and INF $\gamma$, with the overall effect of the reduction of the proliferation of lymphocytes $[39,40]$. However, cyclosporine not only interferes with the immune system, but also has a direct effect on the cytoskeleton of the podocyte. The mechanism involves the sinaptopodina that plays a key role in stabilizing the actin cytoskeleton in podocytes. When the sinaptopodina is phosphorylated, binds to the protein 14-3-3 and is protected from degradation. The calcineurin binds to this complex by starting to degradation; cyclosporine, calcineurin binding, blocks this process [41]. Thus, the antiproteinuric effect of this medication may also be unrelated to the immunosuppressive effects on $\mathrm{T}$ lymphocytes. These findings may provide a rationale for the use in proteinuric non- immune-mediated diseases, such as focal segmental glomerulosclerosis and Alport's disease, in which is used the drug $[1,42]$. Cyclosporin is very dreaded for its side effects, even if the doses used in the course of the glomerulus nephritis are quite low. However, one must always consider the nephrotoxicity of the drug, due in part to vasoconstriction but also to tubular damage. Furthermore, the increase of expression of the TGF-, molecule capable of activating the processes of fibrosis, which is observed in the course of therapy with cyclosporin has an important role in the evaluation of renal fibrosis in the course of the follow- up of the patient [1,43]. Compared with other immunosuppressive drugs, cyclosporine has not myelosuppressive activity that facilitates further use in regimens where it is associated with other immunosuppressants. Among the side effects, you may experience high levels of blood pressure due to increased sensitivity to sodium chloride. It should also be noted that the use of cyclosporine A is associated with increased plasma levels of potassium and uric acid. Furthermore, because the drug is catabolized in the liver by cytochrome P450, common pathway of catabolism of many other molecules, it is important monitoring the plasma levels during simultaneous use of multiple medications. Adverse drug reactions can include gingival hyperplasia, convulsions, peptic ulcers, pancreatitis, fever, vomiting, diarrhea, confusion, hypercholesterolemia, dyspnea, numbness and tingling particularly of the lips, pruritus, burning sensations at finger tips and an increased vulnerability to opportunistic fungal and viral infections. Another drug that can inhibit cyclophilin is tacrolimus that present the same mechanism of action of cyclosporine. Its use is restricted to non-responders to other therapies. Patients treated with the cyclosporine and tacrolimus are at high risk of developing renal injury. Nephrotoxicity is manifested either as acute azotemia, which is largely reversible after reducing the dose, or as chronic progressive renal disease, which is usually irreversible. Other 
renal effects of the calcineurin inhibitors include tubular dysfunction and, rarely, a hemolytic uremic syndrome [1].

\section{Monoclonal Antibodies}

Since the late 1990s, advances in molecular biology have led to a variety of new treatments to immunologic diseases. The major biologic approaches in clinical use include agents able to:

-Interfere with cytokine function

-Inhibit the "second signal" required for T-cell activation

\section{-Deplete B cells}

The treatment of immune-mediated renal diseases consisted in the use of monoclonal antibodies. Currently several monoclonal antibodies directed against membrane proteins expressed on B cells, against cytokines stimulating $\mathrm{B}$ cells or against complement components are available $[44,45]$. Rituximab is an antibody directed against the CD20 antigen of B lymphocytes and is a chimeric monoclonal antibody, which contains heavy and light chains in the murine variable region and human IgG constant region. This configuration allows to use a smaller amount of murine antibody to prolong the half-life of rituximab $[1,45]$. Once bound to CD20, it causes B cell lysis activating direct cytotoxicity and antibodydependent; the final result is the depletion of CD20 that may persist up to 12 months. The depletion of memory $\mathrm{B}$ cells causes a change in the immune response [46], and in particular, a reduced production of antibodies and cytokines, alteration of the process of antigen presentation to B cells and reduced activity of CD40 with subsequent improvement in the course of the disease. This profound interference of rituximab in immune processes, through the depletion of B cells, represents the rationale for its use in immune-mediated diseases. The drug is indicated for the treatment of non-Hodgkin lymphoma and chronic lymphocytic leukemia. Subsequently, it was recognized for the treatment of rheumatoid arthritis and vasculitis, and then its use has been indicated for other diseases. In nephrology rituximab has been used in some of both primitive and secondary glomerulonephritis and in the prevention of transplant rejection [47-49]. Membranous glomerulonephritis is one the most common causes of the nephrotic syndrome in non-diabetic adults and its treatment is still controversial. The primary regimens used to treat idiopathic membranous glomerulonephritis include a cytotoxic drug (cyclophosphamide or, less often, chlorambucil) and glucocorticoids or calcineurin inhibitors with or without glucocorticoids [50]. Relevant interest exists for the results of the treatment with rituximab directed primarily at reducing proteinuria and nephrotic syndrome. At the moment there are some trials that describe how a significant number of patients with membranous nephropathy may benefit from the treatment with rituximab [51]. The nephrotic syndrome, steroid-dependent or steroid-resistant due to glomerular injury in minimal or focal segmental glomerular sclerosis is another condition in which rituximab was used. In cases of nephrotic syndrome steroid-dependent rituximab can be used to avoid high doses of cortisone that are associated with serious side effects (obesity, diabetes, osteoporosis, aseptic necrosis of the femoral head, etc.) and the described results seem promising [51]. The use of rituximab was followed by a remission of nephrotic syndrome in cases of nephrotic syndrome resistant to steroids and immunosuppressants. Systemic lupus erythematosus is characterized by the production of a large number of auto-antibodies that determine a systemic disease caused by the deposition of antigen-antibody immune complexes in various organs.
The treatment of lupus nephritis is based on the use of immunosuppressive drugs in various combinations with variable dose depending on the severity of the lesions (classes histological classification according to the ISN / RPS). On the other hand, despite all the drugs available, there are some patients who do not respond to therapies and who progress in the severity of their clinical condition [45]. Therefore, in this group of patients, it seemed appropriate the use of Rituximab, based on the most recent evidences [48,49]. However, because of the great variability of the lupus disease and the absence of large controlled trials, clear indications for rituximab in lupus are still lacking. The forms of vasculitis associated with the presence of ANCA both P-ANCA and C-ANCA are also other conditions where Rituximab can be used. Rituximab was effective as cyclophosphamide in inducing remission of the acute phase but it was also very effective in the treatment of refractory and/or recurrent forms. The mixed cryoglobulinemia syndrome is generally a disease mediated by the formation of monoclonal IgM (rheumatoid factor) polyclonal IgG determing the clinical manifestations associated with renal and nonrenal involvement [26]. The mixed cryoglobulinemia syndrome is most often induced by hepatitis $\mathrm{C}$ virus (HCV) infection; therefore it has been proposed interferon therapy in order to eradicate the infectious agent. However, It can also be associated with autoimmune or lymphoproliferative disorders or, rarely, can be idiopathic, hence, it was also attempted therapy with rituximab with the intent to reduce the production of cryoglobulins by the depletion of lymphocytes. Rituximab for different schemes have been proposed: $375 \mathrm{mg} / \mathrm{m}^{2}$ for four consecutive weeks or $1 \mathrm{~g}$ every 2 weeks; others suggest that the therapeutic effect should be assessed with CD20 lymphocyte counts. Moreover, there are protocols on when to use rituximab, alone or in combination with steroids or with immune suppressive drugs. Another issue is the safety of these drugs both in the short term, including the risk of infections (leucoencephalitis) and in the longterm with possible negative effects still unknown [45,52].

\section{Anti-Cytokines Therapy}

Understanding the contribution of cytokines to the pathogenesis of immunologic diseases offers novel and creative therapeutic options that were not previously available. Although biologic agents that regulate cytokines showed positive results $[26,45,52]$, small molecules that block cytokine production and/or signaling will likely have a competitive advantage in the next years. An important note of caution, however, regards the possibility that suppressing even physiologic concentrations of some cytokines could have serious adverse effects on immune surveillance. The anti- cytokine therapy is based on the use of drugs directed against the pro-inflammatory cytokines or that promote the action of anti-inflammatory cytokines [13].

\section{Strategies to Inhibit Cytokine Activity}

\section{Antibodies}

A leading approach to neutralize cytokines is the use of specific antibodies directed against the cytokines or its corresponding receptor. Antibodies have several central effects that are important in interfering with cytokine actions. These include excellent solubility, exquisite specificity, and a long half-life in serum [53]. 


\section{Immunoadhesins}

Another approach used to develop biologic inhibitors of cytokine activity is to engineer a fusion protein that combines the constant domains of an antibody molecule with the ligand-recognition domain of a cytokine receptor. Such fusion proteins, or immunoadhesins, can be expressed and secreted from mammalian cells as bivalent proteins that, much the same as immunoglobulins, consist of two polypeptide chains that are disulfide linked [54]. The advantages of this approach are that it not only eliminates the need to immunize an animal, but also circumvents screening for cytokine-specific antibodies: "antigen recognition" is provided by the cytokine receptor that is engineered into the immunoadhesin. Immunoadhesins in general share the extended half-life in serum observed for whole antibodies, and because they are derived from human proteins, they tend to induce immunogenicity [13].

\section{Natural Cytokine Antagonists}

Cytokines expressed during an inflammatory response are tightly controlled by transcriptional and translational levels, and they appear to act locally in that they are cleared rapidly once they diffuse into the vascular compartment. Mechanisms have also evolved to allow rapid neutralization of excess cytokine at the site of inflammation, such as the expression of soluble cytokine-binding proteins found in tissue and blood. Other mechanisms for regulating cytokine-induced pathways include natural antagonists. In the case of interleukin (IL)-1, a soluble form of IL-1 has been described that can bind with high affinity to the cell surface receptor without activating signaling [55]. This protein, called IL-1 receptor antagonist, and marketed as a recombinant biologic called Kineret ${ }^{\oplus}$ (anakinra) competes with IL-1 for binding to receptor [56].

\section{Anti- PDGF}

In experimental GN with mesangial proliferation induced by antibodies directed against an antigen of mesangial cells (anti -Thy 1.1) interesting results were obtained using an aptamer DNA bound to PEG designed to inhibit the action of PDGF $[57,58]$. Two daily doses of this aptamer induced a $95 \%$ reduction of proliferation of mesangial cells typical model GN by anti -Thy 1.1. Recently, the results were confirmed with use of recombinant human monoclonal antibodies directed against PDGF $[13,59]$.

\section{Anti- MCP- 1}

Another molecule that plays a key role in GN is in the acute phase of glomerular exudation and in the chronic phase with interstitial fibrosis is MCP- 1. Considerable interest has turned to therapeutic approach directed against this chemokine [60].

\section{Anti -TGF - ß1}

TGF $-\beta 1$ is certainly the most well-known profibrotic mediator glomerular and tubulointerstitial. Have been tried several ways of blocking the synthesis or activity of TGF - $B 1$ in both primitive and secondary renal diseases $[13,58]$.

\section{Anti - TNF}

These drugs have proven useful in experimental rapidly progressive GN, where they inhibited the formation of crescents [61-67], and in mouse strains having developed spontaneously SLE [68].

\section{Anti-IL- 1}

Very interesting is the ability to simultaneously inhibit TNF and IL-1, then it is because most of the two cytokines produced by monocytes-macrophages and mesangial cells during activation by immune-mediated GN [67]. At present, the combined use of the two drugs was too burdened by side effects $[13,58,59]$.

\section{Use of Anti-inflammatory Cytokines}

Another approach theoretically very interesting is the use of cytokines that act naturally against the inflammatory process, such as IL -4, IL - 10 , IL-13 or modest doses of TG - $ß 1[58,67,68]$.

\section{Plasmapheresis}

This technique enables to remove from the plasma some components, including immunoglobulins and cytokines by extracorporeal circulation $[69,70]$. Therefore, plasmapheresis is indicated in the immune-mediated diseases in which there is a factor known pathogen, such as anti-basement membrane in the course of Goodpasture's disease or anti-metal proteinase (ADAMTS) of thrombotic microangiopathy. The therapeutic apheresis is able to remove solutes circulating pathogens, but also to act on the reticuloendothelial system through an immunomodulating effect. The current indications plasmapheresis have been defined and periodically re-established by two American scientific associations: the American Association of Blood Banks (AABB) and the American Society of apheresis (ASFA), based on the available evidence of efficacy of treatment in specific diseases [71-73].

- Category I, in which the treatment is first choice,

- Category II, in which plasmapheresis has a supporting role,

- Category III, where there are clear demonstrations of usefulness of the technique,

- Category IV, where plasmapheresis is not effective.

Caterogory I, includes Goodpasture's syndrome and thrombotic thrombocytopenia purpura. Category II: cryoglobulinemia, glomerulonephritis rapidly progressive and the' Acute renal failure due to myeloma kidney, Category III: focal segmental glomerulosclerosis applicant, the hemolytic-uremic syndrome, vasculitis, and systemic lupus erythematosus. Category IV: lupus nephritis, renal transplant rejection and the amyloidosis $[1,74,75]$.

\section{Apheresis and nanotechnology}

The therapeutic plasmapheresis has undergone a significant change due to the development of the equipment and to an expansion of the indications. In fact, technological innovation has introduced methods that allow treatment more tolerable and less invasive. In this perspective fits Hemofenix technology and filter ROSA [76]. In contrast to the traditional systems of plasmapheresis by centrifugation, the device Hemofenix uses a system of nanofiltration. Hemofenix allows performing the treatment with a single and small needle and with a reduced extracorporeal volume, about $70 \mathrm{ml}$, reducing the risks 
for the patient. Additional advantages are represented by: low priming and short duration of treatment, furthermore, the exchange of low volumes during the treatment allows you to not use plasma as replacement fluid reducing the risk of infection and allergic reactions present with plasmapheresis [77]. Clinical trials should be performed to demonstrate the effectiveness and safety of this method by comparing this with the traditional apheretic therapy.

\section{Materials and Methods}

We conducted a systematic review on the current knowledge about pathogenetic mechanisms of glomerulonephritis and related therapies.

\section{Types of studies}

RCTs and quasi-RCTs evaluating the current knowledge about pathogenetic mechanisms of glomerulonephritis and related therapy were considered.

\section{Electronic searches}

\section{Cochrane Renal Group's specialised register}

2. AEGIS database (AIDS Education Global Information System (http://www.aegis.com)).

3. ClinicalTrial.gov (http://www.clinicaltrials.gov).

4. WHO International Clinical Trials Registry Platform (http:// www.who.int/ictrp/en/)

\section{MEDLINE}

We checked the reference lists of nephrology text books, review articles, and relevant studies.

\section{Results}

We have summarised the results of the research in Figure 1.

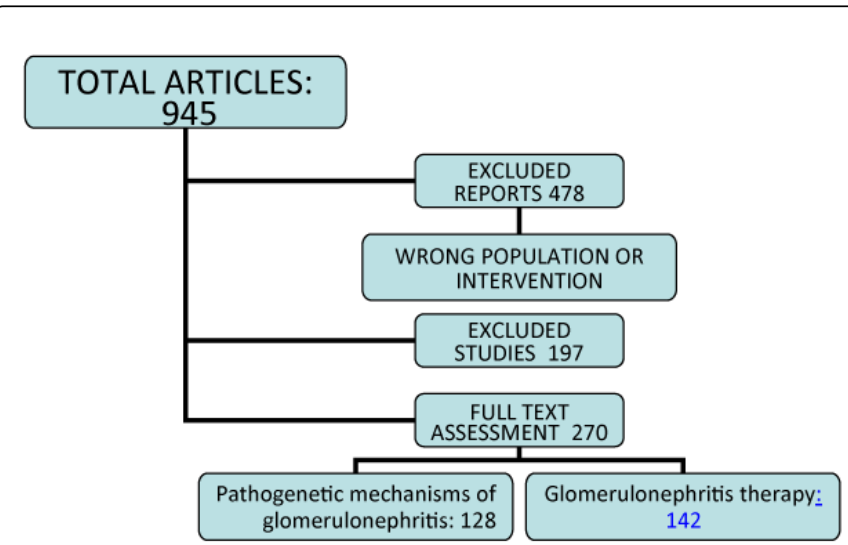

Figure 1: Results of the research.

\section{Conclusion}

The pathogenetic mechanism of glomerulonephritis are numerous and complex, involving both humoral and cell-mediated immune responses. The nature of the immune response leading to GN, is strongly influenced by immunogenetic phenotypes. There were several clinical trials conducted to identify the correct treatment of glomerulonephritis, but currently, although significant advancements over the last 10 years were achieved, important questions remain unanswered. Indeed, we still do not know the mechanisms underlying the absence of clinical responses of some of most of the therapies for glomerulonephritis. Therefore it would be desirable the development of a web enabled database to assist nephrologists during the treatment of patients with glomerulonephritis.

\section{References}

1. Polci R, Feriozzi S (2011) [Immunotherapy mechanisms in glomerulonephritis]. G Ital Nefrol 28: 612-621.

2. Couser WG (1998) Pathogenesis of glomerular damage in glomerulonephritis. Nephrol Dial Transplant 13 Suppl 1: 10-15.

3. Couser WG, Baker PJ, Adler S (1985) Complement and the direct mediation of immune glomerular injury: a new perspective. Kidney Int 28: 879-890.

4. Nangaku M, Alpers CE, Pippin J, Shankland SJ, Adler S, et al. (1997) A new model of renal microvascular endothelial injury. Kidney Int; 52: 182-199

5. Brady HR (1994) Leukocyte adhesion molecules and kidney diseases. Kidney Int 45: 1285-1300.

6. Bakris GL, Weir MR, Shanifar S, Zhang Z, Douglas J, et al. (2003) Effects of blood pressure level on progression of diabetic nephropathy: results from the RENAAL study. Arch Intern Med 163: 1555-1565.

7. Jafar TH, Stark PC, Schmid CH, Landa M, Maschio G, et al. (2003) AIPRD Study Group. Progression of chronic kidney disease: the role of blood pressure control, proteinuria, and angiotensin-converting enzyme inhibition: a patient-level meta-analysis. Ann Intern Med 139: 244-252

8. Nakao N, Yoshimura A, Morita H, Takada M, Kayano T, et al. (2003) Combination treatment of angiotensin-II receptor blocker and angiotensin- converting- enzyme inhibitor in non-diabetic renal disease (COOPERATE): a randomized controlled trial. Lancet 361: 117-124

9. Ruggenenti P, Perna A, Remuzzi G; GISEN Group Investigators (2003) Retarding progression of chronic renal disease: the neglected issue of residual proteinuria. Kidney Int 63: 2254-2261.

10. Ponticelli C, Altieri P, Scolari F, Passerini P, Roccatello D, et al. (1998) A randomized study comparing methylprednisolone plus chlorambucil versus methylprednisolone plus cyclophosphamide in idiopathic membranous nephropathy. J Am Soc Nephrol 9: 444-450.

11. Hiraoka M, Tsukahara $\mathrm{H}$, Matsubara $\mathrm{K}$, Tsurusawa $\mathrm{M}$, Takeda $\mathrm{N}$, et al. (2003) A randomized study of two long-course prednisolone regimens for nephrotic syndrome in children. Am J Kidney Dis 41: 1155-1162.

12. Pozzi C, Andrulli S, Del Vecchio L, Melis P, Fogazzi GB, et al. (2004) Corticosteroid effectiveness in IgA nephropathy: long-term results of a randomized, controlled trial. J Am Soc Nephrol 15: 157-163.

13. Coppo R, Menè $P$ (2004) [Glomerulonephritis and renal sclerosis: new therapeutic proposals (review)]. G Ital Nefrol 21: 217-224.

14. Buttgereit F, Straub RH, Wehling M, Burmester GR (2004) Glucocorticoids in the treatment of rheumatic diseases: an update on the mechanisms of action. Arthritis Rheum 50: 3408-3417.

15. Nagaich AK, Rayasam GV, Martinez ED, Becker M, Qiu Y, et al. (2004) Subnuclear trafficking and gene targeting by steroid receptors. Ann N Y Acad Sci 1024: 213-220.

16. McKay LI, Cidlowski JA (1999) Molecular control of immune/ inflammatory responses: interactions between nuclear factor-kappa B and steroid receptor-signaling pathways. Endocr Rev 20: 435-459.

17. De Bosscher K, Vanden Berghe W, Haegeman G (2003) The interplay between the glucocorticoid receptor and nuclear factor-kappaB or activator protein-1: molecular mechanisms for gene repression. Endocr Rev 24: 488-522.

18. Hafezi-Moghadam A, Simoncini T, Yang Z, Limbourg FP, Plumier JC, et al. (2002) Acute cardiovascular protective effects of corticosteroids are mediated by non-trascriptional activation of endothelial nitric oxide synthase. Nat Med 8: 473-479. 
19. Janeway CA, Travers P, Walport M, Shlomchik MJ (2001) Manipulation of the Immune Response. In: Immunobiology, 5th edition. New York: Gardland Science (NCBI Bookshelf)

20. Rhen T, Cidlowski JA (2005) Antiinflammatory action of glucocorticoids--new mechanisms for old drugs. N Engl J Med 353: 1711-1723.

21. Torrego A, Pujols L, Roca-Ferrer J, Mullol J, Xaubet A, et al. (2004) Glucocorticoid receptor isoforms alpha and beta in in vitro cytokineinduced glucocorticoid insensitivity. Am J Respir Crit Care Med 170: 420-425.

22. Canalis E, Delany AM (2002) Mechanisms of glucocorticoid action in bone. Ann N Y Acad Sci 966: 73-81.

23. Garside H, Stevens A, Farrow S, Normand C, Houle B, et al. (2004) Glucocorticoid ligands specify different interactions with NF-kappaB by allosteric effects on the glucocorticoid receptor DNA binding domain. J Biol Chem 279: 50050-50059.

24. Illei GG, Austin HA, Crane M, Collins L, Gourley MF, et al. (2001) Combination therapy with pulse cyclophosphamide plus pulse methylprednisolone improves long-term renal outcome without adding toxicity in patients with lupus nephritis. Ann Intern Med 135: 248-257.

25. Wilde B, van Paassen P, Witzke O, Tervaert JW (2011) New pathophysiological insights and treatment of ANCA-associated vasculitis. Kidney Int 79: 599-612.

26. Feriozzi S (2012) [Role of monoclonal antibodies in the treatment of immune-mediated kidney disease: an introduction]. G Ital Nefrol 29: 267-273.

27. Hall AG, Tilby MJ (1992) Mechanisms of action of, and modes of resistance to, alkylating agents used in the treatment of haematological malignancies. Blood Rev 6: 163-173.

28. Haubitz M, Bohnenstengel F, Brunkhorst R, Schwab M, Hofmann U, et al. (2002) Cyclophosphamide pharmacokinetics and dose requirements in patients with renal insufficiency. Kidney Int 61: 1495-1501.

29. Takada K, Arefayene M, Desta Z, Yarboro CH, Boumpas DT, et al. (2004) Cytochrome P450 pharmacogenetics as a predictor of toxicity and clinical response to pulse cyclophosphamide in lupus nephritis. Arthritis Rheum 50: 2202-2210.

30. Couser WG (1993) Research opportunities and future directions in glomerular disease. Semin Nephrol 13: 457-471.

31. Tiede I, Fritz G, Strand S, Poppe D, Dvorsky R, et al. (2003) CD28dependent Racl activation is the molecular target of azathioprine in primary human CD4+ T lymphocytes. J Clin Invest 111: 1133-1145.

32. Hiemstra TF, Walsh M, Mahr A, Savage CO, de Groot K, et al. (2010) Mycophenolate mofetil vs azathioprine for remission maintenance in antineutrophil cytoplasmic antibody-associated vasculitis: a randomized controlled trial. JAMA 304: 2381-2388.

33. Houssiau FA, D'Cruz D, Sangle S, Remy P, Vasconcelos C, et al. (2010) Azathioprine versus mycophenolate mofetil for long-term immunosuppression in lupus nephritis: results from the MAINTAIN Nephritis Trial. Ann Rheum Dis 69: 2083-2089.

34. Allison AC, Eugui EM (2000) Mycophenolate mofetil and its mechanisms of action. Immunopharmacology 47: 85-118.

35. Lipsky JJ (1996) Mycophenolate mofetil. Lancet 348: 1357-1359.

36. Appel AS, Appel GB (2009) An update on the use of mycophenolate mofetil in lupus nephritis and other primary glomerular diseases. Nat Clin Pract Nephrol 5: 132-142.

37. Sepe V, Libetta C, Giuliano MG, Adamo G, Dal Canton A (2008) Mycophenolate mofetil in primary glomerulopathies. Kidney Int 73: 154-162.

38. McCune WJ (2005) Mycophenolate mofetil for lupus nephritis. N Engl J Med 353: 2282-2284.

39. Clipstone NA, Crabtree GR (1992) Identification of calcineurin as a key signalling enzyme in T-lymphocyte activation. Nature 357: 695-697.

40. Liu J, Farmer JD Jr, Lane WS, Friedman J, Weissman I, et al. (1991) Calcineurin is a common target of cyclophilin-cyclosporin A and FKBPFK506 complexes. Cell 66: 807-815.
41. Mathieson PW (2008) Proteinuria and immunity--an overstated relationship? N Engl J Med 359: 2492-2494.

42. Cattran DC, Alexopoulos E, Heering P, Hoyer PF, Johnston A, et al. (2007) Cyclosporin in idiopathic glomerular disease associated with the nephrotic syndrome: workshop recommendations. Kidney Int 72: 1429-1447.

43. Islam M, Burke JF Jr, McGowan TA, Zhu Y, Dunn SR, et al. (2001) Effect of anti-transforming growth factor-beta antibodies in cyclosporineinduced renal dysfunction. Kidney Int 59: 498-506.

44. Ponticelli C, Coppo R, Salvadori M (2011) Glomerular diseases and transplantation: similarities in pathogenetic mechanisms and treatment options. Nephrol Dial Transplant 26: 35-41.

45. Feriozzi S (2012) [Role of monoclonal antibodies in the treatment of immune-mediated kidney disease: an introduction]. G Ital Nefrol 29: 267-273.

46. Takei T, Nitta K (2011) Rituximab and minimal change nephrotic syndrome: a therapeutic option. Clin Exp Nephrol 15: 641-647.

47. Salama AD, Pusey CD (2006) Drug insight: rituximab in renal disease and transplantation. Nat Clin Pract Nephrol 2: 221-230.

48. Ramanath V, Nistala R, Chaudhary K (2012) Update on the role of rituximab in kidney diseases and transplant. Expert Opin Biol Ther 12: 223-233.

49. Jayne D (2010) Role of rituximab therapy in glomerulonephritis. J Am Soc Nephrol 21: 14-17.

50. Cattran DC, Treatment of idiopathic membranous nephropathy in UpToDate.

51. Falk RJ, Schur PH, Appel GB (2012) Therapy of diffuse or focal proliferative lupus nephritis in UpToDate.

52. Flamm S, Chopra S, Kaplan A, Appel GB (2012) Treatment of essential cryoglobulinemia in UpToDate.

53. Song XY, Torphy TJ, Griswold DE, Shealy D (2002) Coming of age: anticytokine therapies. Mol Interv 2: 36-46.

54. Engelmann H, Holtmann H, Brakebusch C, Avni YS, Sarov I, et al. (1990) Antibodies to a soluble form of a tumor necrosis factor (TNF) receptor have TNF-like activity. J Biol Chem 265: 14497-14504.

55. Arend WP, Malyak M, Guthridge CJ, Gabay C (1998) Interleukin-1 receptor antagonist: role in biology. Annu Rev Immunol 16: 27-55.

56. Chamow SM, Ashkenazi A (1996) Immunoadhesins: principles and applications. Trends Biotechnol 14: 52-60.

57. Floege J, Ostendorf T, Janssen U, Burg M, Radeke HH, et al. (1999) Novel approach to specific growth factor inhibition in vivo: antagonism of platelet-derived growth factor in glomerulonephritis by aptamers. Am J Pathol 154: 169-179.

58. Iain B McInnes. Cytokine networks in rheumatic diseases: Implications for therapy.

59. Ostendorf T, van Roeyen CR, Peterson JD, Kunter U, Eitner F, et al. (2003) A fully human monoclonal antibody (CR002) identifies PDGF-D as a novel mediator of mesangioproliferative glomerulonephritis. J Am Soc Nephrol 14: 2237-2247.

60. Fujinaka H, Yamamoto T, Takeya M, Feng L, Kawasaki K, et al. (1997) Suppression of anti-glomerular basement membrane nephritis by administration of anti-monocyte chemoattractant protein-1 antibody in WKY rats. J Am Soc Nephrol 8: 1174-1178.

61. Isaka Y, Brees DK, Ikegaya K, Kaneda Y, Imai E, et al. (1996) Gene therapy by skeletal muscle expression of decorin prevents fibrotic disease in rat kidney. Nat Med 2: 418-423.

62. Zhou A, Ueno H, Shimomura M, Tanaka R, Shirakawa T, et al. (2003) Blockade of TGF-beta action ameliorates renal dysfunction and histologic progression in anti-GBM nephritis. Kidney Int 64: 92-101.

63. Daniel C, Takabatake Y, Mizui M, Isaka Y, Kawashi H, et al. (2003) Antisense oligonucleotides against thrombospondin-1 inhibit activation of tgf-beta in fibrotic renal disease in the rat in vivo. Am J Pathol 163: 1185-1192.

64. Huang Y, Haraguchi M, Lawrence DA, Border WA, Yu L, et al. (2003) A mutant, noninhibitory plasminogen activator inhibitor type 1 decreases 
Citation: Russo GE, Musto TG, Testorio M, Molfino A, Martinez A, et al. (2014) Glomerulonephritis, Pathogenetic Mechanisms and Therapeutic Options: An Overview. J Nephrol Ther 4: 175. doi:10.4172/2161-0959.1000175

Page 9 of 9

matrix accumulation in experimental glomerulonephritis. J Clin Invest 112: 379-388.

65. Benigni A, Zoja C, Corna D, Zatelli C, Conti S, et al. (2003) Add-on antiTGF-beta antibody to ACE inhibitor arrests progressive diabetic nephropathy in the rat. J Am Soc Nephrol 14: 1816-1824.

66. Isaka Y, Tsujie M, Ando Y, Nakamura H, Kaneda Y, et al. (2000) Transforming growth factor-beta 1 antisense oligodeoxynucleotides block interstitial fibrosisin unilateral ureteral obstruction. Kidney Int 58 : 1885-1892.

67. Karkar AM, Koshino Y, Cashman SJ, Dash AC, Bonnefoy J, et al. (1992) Passive immunization against tumour necrosis factor-alpha (TNF-alpha) and IL-1 beta protects from LPS enhancing glomerular injury in nephrotoxic nephritis in rats. Clin Exp Immunol 90: 312-318.

68. Edwards CK 3rd, Zhou T, Zhang J, Baker TJ, De M, et al. (1996) Inhibition of superantigen-induced proinflammatory cytokine production and inflammatory arthritis in MRL-lpr/lpr mice by a transcriptional inhibitor of TNF-alpha. J Immunol 157: 1758-1772.

69. Smith JW, Weinstein R, Hillyer KL; AABB Hemapheresis Committee; American Society for Apheresis (2003) Therapeutic apheresis: a summary of current indication categories endorsed by the $\mathrm{AABB}$ and the American Society for Apheresis. Transfusion 43: 820-822.

70. Malchesky PS, Koo AP, Skibinski CI, Hadsell AT, Rybicki LA (2010) Apheresis technologies and clinical applications: the 2007 International Apheresis Registry. Ther Apher Dial 14: 52-73.
71. Szczepiórkowski ZM, Bandarenko N, Kim HC, et al. Guidelines on the use of therapeutic apheresis in clinical practice: evidence-based approach from the Apheresis Applications Committee of the American Society for Apheresis. J Clin Apher 2007; 22: 106-75.

72. Szczeklik W, Mitka I, Nowak I, Seczyńiska B, Sega A, et al. (2010) [Plasmapheresis in intensive therapy unit]. Anestezjol Intens Ter 42: 100-106.

73. Schwartz J, Winters JL, Padmanabhan A, Balogun RA, Delaney M, et al. (2013) Guidelines on the use of therapeutic apheresis in clinical practiceevidence-based approach from the Writing Committee of the American Society for Apheresis: the sixth special issue. J Clin Apher 28: 145-284.

74. Russo GE, Bauco B, De Paola A, Vitaliano E, Bonello M (2000) Principali campi di applicazione dell'Aferesi Terapeutica. Atti V Corso di Aggiornamento in Emaferesi ed Autotrasfusione. In: Progressi in Emaferesi. Edizioni S.I.d.E.S, Roma, 63-74.

75. Russo GE e coll.: Therapeutical apheresis: cost analysis. The International Journal of Artificial Organs, vol 21, S-6, pp 72-77, 1998.

76. VoÄnov AV, Bedrov AIa, Voinov VA (2012) [Syndrome of "diabetic foot"]. Vestn Khir Im I I Grek 171: 106-109.

77. Voinov VA (2013) [Strategy of efferent therapy in sepsis]. Vestn Khir Im I I Grek 172: 74-77. 